\title{
Comparative transcriptome analyses of different Salvia miltiorrhiza varieties during the accumulation of tanshinones
}

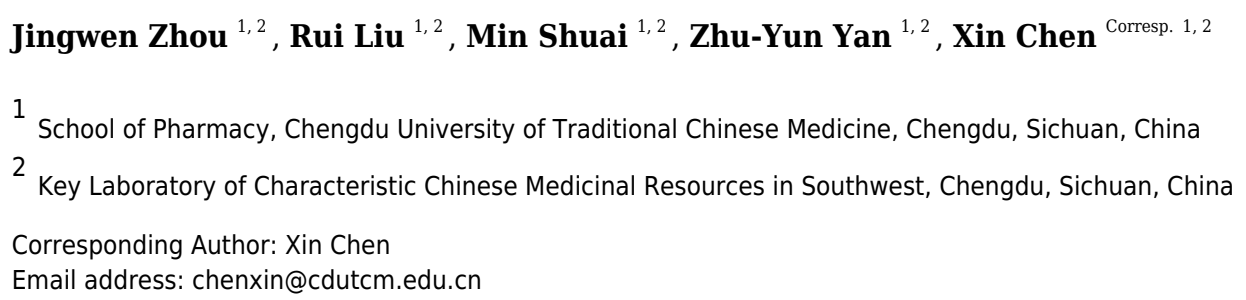

Salvia miltiorrhiza (Labiatae) is an important medicinal plant in traditional Chinese medicine. Tanshinones are one of the main active components of $S$. miltiorrhiza. It has been found that the intraspecific variation of $S$. miltiorrhiza is relatively large and the content of tanshinones in its roots of different varieties is also relatively different. To investigate the molecular mechanisms that responsible for the differences among these varieties, the tanshinones content was determined and comparative transcriptomics analysis was carried out during the tanshinones accumulation stage. A total of 52,216 unigenes were obtained from the transcriptome by RNA sequencing among which 23,369 genes were differentially expressed among different varieties, and 2,016 genes including 18 diterpenoid biosynthesis-related genes were differentially expressed during the tanshinones accumulation stage. Functional categorization of the differentially expressed genes (DEGs) among these varieties revealed that the pathway related to photosynthesis, oxidative phosphorylation, secondary metabolite biosynthesis, diterpenoid biosynthesis, terpenoid backbone biosynthesis, sesquiterpenoid and triterpenoid biosynthesis are the most differentially regulated processes in these varieties. The six tanshinone components in these varieties showed different dynamic changes in tanshinone accumulation stage. In addition, combined with the analysis of the dynamic changes, 277 DEGs (including 1 dehydrogenase, 3 CYP450 and 24 transcription factors belonging to 12 transcription factor families) related to the accumulation of tanshinones components were obtained.

Furthermore, the KEGG pathway enrichment analysis of these 277 DEGs suggested that there might be an interconnection between the primary metabolic processes, signaling processes and the accumulation of tanshinones components. This study expands the vision of intraspecific variation and gene regulation mechanism of secondary metabolite biosynthesis pathways in medicinal plants from the "omics" perspective. 


\section{Comparative transcriptome analyses of different Salvia}

\section{2 miltiorrhiza varieties during the accumulation of tanshinones}

3 Jingwen Zhou ${ }^{1,2}$, Rui Liu ${ }^{1,2}$, Min Shuai ${ }^{1,2}$, Zhu-yun Yan ${ }^{1,2}$, Xin Chen ${ }^{1,2}$ *

$4{ }^{1}$ School of Pharmacy, Chengdu University of Traditional Chinese Medicine, Chengdu, Sichuan, China

$5{ }^{2}$ Key Laboratory of Characteristic Chinese Medicinal Resources in Southwest China, Chengdu, Sichuan,

6 China

7

8

9

10

11 Corresponding Author:

12 Xin Chen ${ }^{1,2}$

13 Email address: chenxin@cdutcm.edu.cn 
18 Comparative transcriptome analyses of different Salvia

19

20

21

22

23

24

25

26

27

28

29

30

31

32

33

34

35

36

37

38

\section{miltiorrhiza varieties during the accumulation of tanshinones}

Jingwen Zhou ${ }^{1,2}$, Rui Liu ${ }^{1,2}$, Min Shuai1 ${ }^{1,2}$, Zhu-yun Yan ${ }^{1,2}$, Xin Chen ${ }^{1,2 *}$

${ }^{1}$ School of Pharmacy, Chengdu University of Traditional Chinese Medicine, Chengdu, Sichuan, China

${ }^{2}$ Key Laboratory of Characteristic Chinese Medicinal Resources in Southwest China, Chengdu, Sichuan, China

Corresponding Author:

Xin Chen ${ }^{1,2}$

Email address: chenxin@cdutcm.edu.cn

\section{Abstract}

Salvia miltiorrhiza (Labiatae) is an important medicinal plant in traditional Chinese medicine.

Tanshinones are one of the main active components of $S$. miltiorrhiza. It has been found that the intraspecific variation of $S$. miltiorrhiza is relatively large and the content of tanshinones in its roots of different varieties is also relatively different. To investigate the molecular mechanisms that are responsible for the differences among these varieties, the tanshinones content was determined and comparative transcriptomics analysis was carried out during the tanshinones accumulation stage. A total of 52,216 unigenes were obtained from the transcriptome by RNA sequencing among which 23,369 genes were differentially expressed among different varieties, and 2,016 genes including 18 diterpenoid biosynthesis-related genes were differentially expressed during the tanshinones accumulation stage. Functional categorization of the differentially expressed genes (DEGs) among these varieties revealed that the pathway related to photosynthesis, oxidative phosphorylation, secondary metabolite biosynthesis, diterpenoid biosynthesis, terpenoid backbone biosynthesis, sesquiterpenoid and triterpenoid biosynthesis are the most differentially regulated processes in these varieties. The six tanshinone components in these varieties showed different dynamic changes in tanshinone accumulation stage. In addition, combined with the analysis of the dynamic changes, 277 DEGs (including 1 dehydrogenase, 3 CYP450 and 24 transcription factors belonging to 12 transcription factor families) related to the 
52 accumulation of tanshinones components were obtained. Furthermore, the KEGG pathway 53 enrichment analysis of these 277 DEGs suggested that there might be an interconnection 54 between the primary metabolic processes, signaling processes and the accumulation of 55 tanshinones components. This study expands the vision of intraspecific variation and gene 56 regulation mechanism of secondary metabolite biosynthesis pathways in medicinal plants from 57 the "omics" perspective.

58

59

60

61

62

63

64

65

66

67

68

69

70

71

72

73 74 (Liang et al. 2015).

75

76

77 78

\section{Introduction}

Salvia miltiorrhiza is a plant of the genus Salvia in the family Labiatae. Its dried roots and rhizomes are called Danshen in China, and are often used to treat cardiovascular disease (Dan et al. 2019) and Alzheimer's disease (Zhang et al. 2016). Lipophilic tanshinone is one of the main active components of Danshen (Ma 2015), which is derived predominantly from the plastid methylerythritol phosphate (MEP) pathway or partly through the cytoplasmic mevalonate (MVA), and then synthesized through diterpene biosynthesis pathways (Zhang et al. 2015), including tanshinone I, tanshinone IIA, tanshinone IIB, cryptotanshinone, and dihydrotanshinone, which are mainly accumulated in the roots and rhizomes of S. miltiorrhiza (Chang et al. 2019). As far as the general growth trend of S. miltiorrhiza is concerned, it can be divided into three stages. The first stage is the vegetative growth period in which the aerial part of S. miltiorrhiza grows rapidly. The second stage is the flowering phase, from bolting to flower withering. The third stage is the post-anthesis phase, from the end of flowering to the second vegetative growth period. Previous studies have shown that tanshinones contents began to accumulate in the post-anthesis stage and reached a peak after 60 days in the post-anthesis phase

S. miltiorrhiza is widely distributed and cultivated in China. In the long-term adaptation process to the different local growing environments, it has formed rich genetic diversity. Previous studies about the diversity among varieties of $S$. miltiorrhiza from different habitats was performed by using molecular markers such as inter simple sequence repeats (ISSR), sequence-related 
80 amplified polymorphism (SRAP) (Song et al. 2010), amplified fragment length polymorphism

81 (AFLP) (Wang et al. 2007), expressed sequence tag-derived simple sequence repeat markers

82 (EST-SSR) (Deng et al. 2009; Wang et al. 2011), CAAT box polymorphism (CBDP) (Fabriki-

83 Ourang \& Karimi 2019) and random amplified polymorphism-DNA (RAPD) (Sunar et al. 2020).

84 With the rapid development of sequencing technologies, genome sequencing of some $S$.

85 miltiorrhiza varieties has been carried out by Zhang et al. (2015), Xu et al. (2016), and Song et

86 al. (2020). The transcriptome technique is mainly used for the study of the inducing effect (Gao

87 et al. 2014; Ge et al. 2015), tissue-specific expression (Yang et al. 2013), and discovering the key

88 enzyme genes and transcription factors (TFs) (Song et al. 2017; Zhan et al. 2019) involved in

89 tanshinones and phenolic acids biosynthesis in S. miltiorrhiza. The comparative transcriptomics

90 technique can be used for understanding the differential expression of genes in specific tissues at

91 specific stages, discovering genes related to specific physiological functions, and inferring the

92 physiological functions of unknown genes.

93

94 Secondary metabolites of plants are very important for adaptation, and are products of response

95 to environmental stress. Therefore, there is great variation in the type and/or content of

96

97

98

99

100

101

102

103

104

105

106 secondary metabolites (Isah 2019; Yang et al. 2018). Studies have shown that secondary

metabolites vary among related species or individuals in the same species, which are related to

transcriptome changes and are heritable ( $\mathrm{Li}$ et al. 2020). The regulation mechanisms of the

biosynthetic pathways and transcriptome of plant secondary metabolism have been studied in

many closely related species or different varieties of same species. For example, a comparative

transcriptome analysis of two varieties of Andrographis paniculata (Burm. f.) Nees. with

significant differences in diterpene andrographolide contents revealed that the expression levels of genes related to the biosynthesis of secondary metabolite andrographolide were higher in the genotype with high andrographolide content as compared to that in the low content group (Patel et al. 2020). Matricaria recutita and Chamaemelum nobile are two chamomile species with different chemical compositions, the comparative analysis of their transcriptome revealed that

Peer] reviewing PDF | (2021:03:58666:4:0:NEW 10 Sep 2021) 
107 different gene regulation in the synthesis of terpenoid may contribute to the variation in terpenes 108 types and concentrations in these two species. Combining transcriptome analysis with the results 109 of the content and composition of essential oil terpenoids therein can help to understand the 110 mechanisms of differential terpenoid accumulation in different chemotypes of Cinnamomum 111 camphora (Chen et al. 2018a). The metabolomics study of S. miltiorrhiza found that the same 112 variety grown in different places and varieties of S. miltiorrhiza grown in the same place had 113 significant variation in their metabolome, and the contents of bioactive components were 114 affected by the growing environment and genotype (Zhao et al. 2016). However, there is a lack 115 of comparative analysis based on transcriptome among different varieties of S. miltiorrhiza under 116 the same cultivation environment.

118 An in-depth study on the differences in transcriptional regulation among different S. miltiorrhiza 119 varieties can help to elucidate the ecological adaptation mechanism and the regulation 120 mechanism of tanshinones biosynthesis. In this study, S. miltiorrhiza from typical production areas were cultivated in the same experimental field for six years. Their tanshinones content was determined, then four varieties with significant differences in tanshinones content were selected for subsequent transcriptome analysis. Transcriptional changes during tanshinones accumulation stage were compared while the four varieties were grown under the same environmental conditions, allowing us to investigate the relationship between gene expression regulation and tanshinones accumulation in different varieties of S. miltiorrhiza.

\section{Materials \& Methods}

Plant material and RNA sample collection

130 The selected four varieties of S. miltiorrhiza were collected from Sichuan (SC), Yunnan (YN), 131 Shaanxi (SX), and Henan (HN) (Table 1). All the varieties were asexually propagated through 132 root cutting and cultivated in the experimental field of Chengdu University of Traditional 133 Chinese Medicine for six years. Roots were sampled at 2 days post-anthesis (S1, the early stage 
134 of tanshinones accumulation) and 60 days post-anthesis (S2, the late stage of tanshinones 135 accumulation). Each sample had three biological replicates and was frozen in liquid nitrogen as 136 soon as sampled and then stored at $-80^{\circ} \mathrm{C}$ for subsequent tanshinones contents determination and 137 transcriptome analysis.

138

\section{Determination of tanshinones contents in $S$. miltiorrhiza roots}

140 The contents of tanshinones in four varieties of $S$. miltiorrhiza roots at two periods were

141 determined using ultra-performance liquid chromatography. Tanshinones content was

142 determined on an Agilent 1290 Infinity UPLC system equipped with a diode array detector

143 (DAD, G4212A) using a ZORBAX Eclipse Plus C18 Rapid Resolution HD HPLC column

$144(2.1 \times 50 \mathrm{~mm}, 1.8 \mathrm{um}$, Agilent). The mobile phase consisted of $0.02 \%$ (vol/vol) phosphoric acid

145 solution (A) and acetonitrile (B) at a flow rate of $0.4 \mathrm{~mL} \cdot \mathrm{min}^{-1}$. The UPLC chromatogram was

146 monitored at $270 \mathrm{~nm}$ and the column temperature was set at $25^{\circ} \mathrm{C}$. The six bioactive

147 compositions including tanshinone IIB (TNIIB), dihydrotanshinone I (DTNI), tanshinone I

148 (TNI), cryptotanshinone (CTN), tanshinone IIA (TNIIA) and miltrinoe (MTN), were compared

149 with the authorized standard (Chengdu Alpha Biotechnology Co., Ltd., China). Total tanshinone 150 in this study was comprised of TNIIB, DTNI, TN1, CTN, TNIIA, and MTN.

151

152 RNA extraction, library preparation, and sequencing

153 Total RNA was extracted using Trizol reagent kit (Invitrogen, Carlsbad, CA, USA) according to 154 the manufacturer's protocol. RNA's integrity was confirmed by using the 2100 Bioanalyzer 155 (Agilent Technologies, Palo Alto, CA, USA) and checked using RNase-free agarose gel 156 electrophoresis. After total RNA was extracted, mRNA was enriched by Oligo(dT) beads. Then 157 the enriched mRNA was fragmented into short fragments using fragmentation buffer and reverse 158 transcripted into cDNA with random primers by using NEBNext Ultra II RNA Library Prep Kit 159 for Illumina (New England Biolabs, USA). Second strand cDNA was synthesized by DNA 160 polymerase I, RNase $\mathrm{H}, \mathrm{dNTP}$ and buffer. Then the cDNA fragments were purified with 
161 QiaQuick PCR purification kit (Qiagen, Germany), end repaired, poly(A) added, and ligated to

162 Illumina sequencing adapters. The ligation products were size selected by agarose gel

163 electrophoresis, PCR amplified, and sequenced using Illumina HiSeq2500 by Gene Denovo

164 Biotechnology Co., Ltd (Guangzhou, China), and 150 bp paired-end reads were generated.

165

166

Data processing, assembly, and annotation

167 Fastp (Chen et al. 2018b) was used to remove sequencing junctions, primers, and low-quality

168 sequences to obtain high-quality clean reads. All downstream analyses were based on high169 quality clean data as determined by Q30, meaning that the error rate was less than $0.1 \%$. High170 throughput sequencing data were de novo assembled using Trinity (Grabherr et al. 2011).

171

172 The unigenes were annotated using BLASTX program against the NCBI non-redundant protein

173 (Nr) database (http://www.ncbi.nlm.nih.gov), the Swiss-Prot protein database

174 (http://www.expasy.ch/sprot), the Kyoto Encyclopedia of Genes and Genomes (KEGG) database

175 (http://www.genome.jp/kegg). When annotating the unigenes with the BLAST program (E-value

176 threshold was set $<10^{-5}$ ), the target sequence with the lowest E-value was selected as the best

177 aligning results. To further assign functions to each unigene, Gene Ontology (GO) annotation

178 was performed by the Blast2GO program according to the categories of Cellular component,

179 Molecular Function, and Biological Process (http://www.geneontology.org/).

180

181 Protein sequences obtained from CDS prediction of unigenes were aligned by Hmmscan

182 (Thiriet-Rupert et al. 2016) to search the domain of the plant transcription factors and these

183 unigenes were classified according to plant TFdb3 to predicted transcription factors (Jin et al.

184 2014).

185

186 Gene expression levels calculation and identification of differentially expressed genes 
187 The assembled unigenes were quantified using RSEM (Li \& Dewey 2011) software, and the 188 results were normalized to Fragments Per Kilobase of transcript sequence per Million base pairs 189 sequenced (FPKM). For each unigene, the threshold of FPKM $>1$ was considered as an 190 expressed gene, and the results could be directly used to compare the differences in gene 191 expression.

192

193 DESeq2 (Love et al. 2014) was used to analysis of significant differential expression in two194 group comparisons. An adjusted $P$-value $<0.05$ and $\mid \log 2$ (FoldChange) $\mid>1$ was set as the 195 threshold for a significant differentially expression level. DEGs among four S. miltiorrhiza 196 varieties at two stages were identified by comparing each pair of varieties (i.e., $\mathrm{HN}-\mathrm{SC}, \mathrm{HN}-\mathrm{SX}$, 197 HN-YN, SX-SC, SX-YN, and YN-SC). Tanshinone accumulation-related DEGs were identified 198 by comparing the genes expression of each variety during accumulation stages (i.e., SC1-SC2, 199 YN1-YN2, SX1-SX2, and HN1-HN2).

200

\section{Statistical Analysis}

$202 \mathrm{R}$ (version 3.5.1) was used for the basic statistical analysis. To evaluate differences of 203 tanshinones content among the four varieties during the post-anthesis stage, Student's t-test was 204 carried out. The two-way ANOVA method was used to analyze the effect of different stages, 205 varieties and interaction between stages and varieties on tanshinones accumulation. Significant 206 differences were shown to be statistically significant $(\mathrm{P}<0.01)$. Spearman's rank correlation 207 coefficient was used to calculate the correlation between gene expression and tanshinone 208 content, and correlation coefficient $>0.70$ was set as a significance level.

209

\section{Validation of RNA-Seq data by quantitative real-time RT-PCR}

211 The validation was performed with the same RNA samples used for RNA-seq analysis. The three 212 biological replicates of the four varieties of S. miltiorrhiza (SC, YN, SX, and YN) at two 213 accumulation stages were analyzed. cDNA synthesis was done by using $400 \mathrm{ng}$ of the total RNA 
214 samples with RT Easy ${ }^{\mathrm{TM}}$ II Kit (Foregene CO., LTD., Chengdu, China). For quantitative PCR, 215 reverse transcribed cDNA products were used as templates. Differentially expressed genes 216 related to tanshinones biosynthesis and candidate transcription factors related to tanshinones 217 accumulation were selected for validation. The primers employed in the qRT-PCR experiments 218 were designed according to the assembled sequences. Primer sequences were reported in Table 219 S1. Actin was used as an endogenous control for the normalization of expression levels of genes 220 (Jiang et al. 2020). The reaction was carried out on a CFX Opus Real-Time PCR System using 221 the Real-Time PCR Easy ${ }^{\mathrm{TM}}$-SYBR Green I (Foregene CO., LTD., Chengdu, China) with a total 222 reaction volume of $20 \mu \mathrm{L}, 0.4 \mu \mathrm{M}$ of the primer, and $80 \mathrm{ng}$ of cDNA. Relative gene expression 223 levels were calculated using the $2^{-\Delta \Delta C t}$ method (Yuan et al. 2006). To ensure reproducibility and 224 reliability, three biological replications and three technical replications were implemented for 225 each sample.

226

\section{Results}

228

229

230

231

232

233

234

235 236

237 238 239 240

\section{Determination of tanshinones contents of different $\boldsymbol{S}$. miltiorrhiza varieties during} tanshinones accumulation

The tanshinones contents of the four S. miltiorrhiza varieties (SC, YN, SX, and $\mathrm{HN}$ ) were measured at different tanshinones accumulation stages (Fig. 1). The results showed that the accumulation of tanshinones in the four S. miltiorrhiza varieties were different at the two stages under the same environmental conditions (Fig. 1). At S1 stage, the tanshinones contents of each variety were low. The contents of tanshinones in all samples was significantly different between S1 and S2 stage $(\mathrm{p}<0.01$, Table S2), and the content of total tanshinone at S2 was about $6.48 \sim$ 20.77 times higher than that at S1. By comparing the tanshinones content in different varieties of S. miltiorrhiza, it was observed that at S2 the content of CTN, TNIIA, and TNI increased by 7.71, 7.88, and 24.01 times respectively. While, compared with at S1, the content of DTNI, TNIIB and MTN increased only by 3.44, 5.00 and 5.42 times at S2. This phenomenon is consistent with the research previous results of Yang L et al. (2013), Chang et al. (2019), 
241 Contreras et al. (2019), and Zhan et al. (2019) in differential tanshinones contents accumulating

242 in varied degrees. The two-way ANOVA of tanshinone showed that both stage and variety had

243 significant effects $(\mathrm{P}<0.01)$ on tanshinones accumulation, and the interaction between them was

244 significant $(\mathrm{P}<0.01)$, indicating that the combination of varieties and growth stages had an

245 impact on the tanshinones accumulation (Table S3).

246

247 Sequencing, assembly, and functional annotation of genes in four varieties of $S$. miltiorrhiza

248 To explore the expression of genes in the four varieties of S. miltiorrhiza during tanshinones 249 accumulation, the transcriptome at tanshinones accumulation stages of the four varieties was 250 analyzed by RNA-Seq. A total of 24 cDNA libraries were constructed from root samples with 251 three biological replicates for each stage and each S. miltiorrhiza variety after removing the 252 adapter reads. The average GC content of the transcriptome was $41.17 \%$. Each sample yielded 253 more than $5.27 \mathrm{~Gb}$ clean data (Table S4). The Q20 and Q30 percentages were more than $92.80 \%$ 254 and $75.22 \%$, respectively. The transcriptome sequencing data from the four S. miltiorrhiza 255 varieties were filtered and assembled by Trinity resulting in a total of 70,357 unigenes. Overall, 256 the unigenes obtained showed a total assembled bases of 75,359,646, a mean length of 1,071 257 base pairs (bp) and a mean N50 of 1,911 bp.

258

259 These assembled unigenes were searched against the NCBI Nr, SwissProt, KEGG, and KOG 260 databases, and it was found that approximately $56.88 \%$ of the unigenes could be annotated in at 261 least one database. The Venn map revealed that 19,454 unigenes were found to be common 262 annotated among four databases (Fig. S1). By sifting out the genes with low expression levels $263(\mathrm{FPKM}<1), 52,216$ genes were obtained (Table S5). KEGG database can link genomic 264 information with functional information, and with KEGG annotation, the functional genes 265 needed for different research can be deeply mined. In this study, a total of 112 genes related to 266 tanshinones biosynthesis were identified, including the terpenoid backbone biosynthesis pathway 267 (Ko00900), diterpenoid biosynthesis pathway (Ko00904) (Table S6). 


\section{Differential gene expression regulation in four $S$. miltiorrhiza varieties}

270 To understand the different regulation of gene expression in different varieties of S. miltiorrhiza 271 during tanshinones accumulation stages, we identified the DEGs between each pair of varieties 272 (i.e., HN-SC, HN-SX, HN-YN, SX-SC, SX-YN, and YN-SC) at S1 and S2 (Fig. 2). Among the 273 pairwise comparison groups, a total of 11368 and 17639 DEGs were detected in S1 and S2 274 stages respectively (Fig. S3). Only one IREG3 (Unigene 0009899) gene was differentially 275 expressed in all the pairwise comparison groups at S1 (Fig. S3A), while there was no common 276 differentially expressed gene at S2 (Fig. S3B). Additionally, the number of DEGs in each 277 pairwise comparison during tanshinones accumulation revealed that the dynamic regulation of 278 gene expression was varied among the four varieties (Table 2). Except for YN-HN and YN-SC, 279 the number of DEGs at S1 was more than that at S2 in the other four pairwise comparison groups 280 (Table 2).

281

282

KEGG enrichment analysis is helpful to further understand the potential function of the genes. In 283 stage S1, DEGs in 6 pairwise comparison groups were mapped to 100 - 128 KEGG pathways (Table S7). Of these KEGG pathways, 88 biological processes related to primary metabolism, 285 secondary metabolism, and signal transduction processes were identified in all pairwise comparison groups (HN1-SC1, HN1-SX1, HN1-YN1, SX1-SC1, SX1-YN1 and YN1-SC1) (Table S7). In stage S2, DEGs in 6 pairwise comparison groups were mapped to 73 - 136 KEGG pathways, and 51 pathways related to secondary metabolism and signal transduction were identified in all pairwise comparison groups (Table S8). Of these KEGG pathways, 48 metabolic processes including plant-pathogen interaction, MAPK signaling pathway, galactose metabolism, pyruvate metabolism, amino sugar and nucleotide sugar metabolism, and oxidative phosphorylation were the identified in all pairwise comparison at the two stages (Table S7). At stage S1, DEGs were specifically enriched in some processes of primary metabolism 
295 mainly related to metabolic processes (thiamine metabolism and propanoate metabolism) and 296 plant circadian regulation (Table S8).

297

298 DEGs related to the changes of tanshinones content during the accumulation of 299 tanshinones

300 To comprehensively understand the regulation of $S$. miltiorrhiza gene expression during the 301 accumulation of tanshinones period, the gene expression of the four varieties in two stages (i.e., 302 SC1-SC2, YN1-YN2, SX1-SX2, and HN1-HN2) were compared. A total of 2016 DEGs 303 containing 18 genes involved in the diterpenoid biosynthesis were obtained (Table S9). Among 304 them, 10 DEGs including HSP TF (Unigene0006797), RBG (Unigene0014447), and GAP3 305 (Unigene0014163) etc. were differentially expressed in all the four pairwise comparison groups 306 (Fig. 3A, Table S9). Compared with stage S1, the expression of these TFs at stage S2 was up307 regulated (Fig. 3B, Fig. 3C). The KEGG enrichment analysis of 2016 DEGs showed that 308 photosynthesis, secondary metabolites biosynthesis, oxidative phosphorylation, diterpenoid 309 biosynthesis, terpenoid backbone biosynthesis, sesquiterpene and triterpene biosynthesis were 310 the most differentially regulated biological processes during the tanshinones accumulation stages 311 among four varieties of S. miltiorrhiza (Fig. 3D).

312

313 To identify the tanshinones accumulation-related genes, the correlation between 2016 DEGs with 314 the significant accumulated components (DTNI, TNIIA, and TNI) was analyzed, respectively. 315 Using this method, 277 DEGs correlated with all the three components were obtained, containing 3161 dehydrogenase gene and 3 CYP450 genes (Table S10, Fig. S4). To understand which categories 317 of these genes were overrepresented, these DEGs were further analyzed by a KEGG enrichment 318 analysis. The KEGG pathway analysis mapped to 63 categories, including photosynthesis, carbon 319 fixation in photosynthetic organisms, galactose metabolism, oxidative phosphorylation, 320 biosynthesis of secondary metabolites, ubiquinone and other terpenoid-quinone biosynthesis, 321 metabolic pathways, MAPK signaling pathway, and plant-pathogen interaction (Table S10). The 
322 top 20 KEGG pathways with the highest representation are shown in Fig. 3E. These results suggest 323 that the accumulation of tanshinones is not only related to the process of tanshinones biosynthesis, 324 but also related to other secondary metabolic processes, signal transduction processes, and even 325 primary metabolic processes.

326

327 Transcription factors (TFs) are a class of proteins that specifically bind to gene promoters and 328 regulate their expression at different levels. In our transcriptome analysis, a total of $1420 \mathrm{TFs}$ 329 was identified, which could be classified to 55 families (Table S11). During the tanshinones 330 accumulation stage, a total of 124 differentially expressed TFs were identified in the four 331 varieties (i.e., SC1-SC2; YN1-YN2; SX1-SX2; HN1-HN2) (Table S12). Among the genes 332 associated with tanshinone accumulation, there are $24 \mathrm{TFs}$, including $6 \mathrm{NAC}, 4 \mathrm{AP} 2 / \mathrm{ERF}, 3$ 333 WRKY, 2 MYB and 1 MYB-related transcription factor (Table S12). These TFs were up334 regulated in all varieties of S. miltiorrhiza at stage S2, and significantly up-regulated in YN2 335 with high tanshinones concentrations (Fig. 3F).

\section{Experimental validation of differential expressed genes by qRT-PCR}

338 To verify the reliability of the RNA-seq results, 10 DEGs at S1 and 10 DEGs at S2 were selected 339 for qRT-PCR analysis (Table S1). These gens included differentially expressed genes related to tanshinones biosynthesis and candidate transcription factors related to tanshinones accumulation

341 (Table S1). The expression levels of these genes were consistent with those determined using 342 RNA-Seq both in stage $\mathrm{S} 1\left(R^{2}=0.8066\right)$ and $\mathrm{S} 2\left(R^{2}=0.8484\right)$ (Fig. 4, Fig. S5), indicating that the 343 dataset obtained by RNA-seq was reliable for gene expression analysis.

344

345 Discussion

346 Besides terpenoid biosynthetic pathway, tanshinones biosynthesis were also associated with 347 primary metabolism, signal transduction and other secondary metabolism processes. A large 348 number of DEGs related to tanshinones accumulation were obtained by the comparative 
349 transcriptomics and the tanshinones content dynamic changes analysis. Functional categorization

350 of these DEGs was enriched in terpenoid biosynthesis, photosynthesis, oxidative

351 phosphorylation, and plant hormone signal transduction (Fig. 3D, Fig. 3E).

352

353 Previous studies have found that the accumulation of secondary metabolites is regulated by 354 cross-talking signaling cascades. Mitogen-activated protein kinase (MAPK) cascades are a

355 prevalent characteristic of eukaryotic cells, involving in plant growth (Zhang et al. 2018),

356 development (Kalapos et al. 2019), stress response (Adachi et al. 2015; He \& Meng 2020), and

357 the biosynthesis of secondary metabolites (De Boer et al. 2011; Devendrakumar et al. 2018;

358 Zheng et al. 2019). In S. miltiorrhiza, eighteen MAPKs have been identified, and two

359 SmMAPKs (SmMAPK1, SmMAPK3) might play a role in tanshinones biosynthesis (Xie et al.

360 2020). Among the DEGs associated with tanshinones accumulation obtained in this study, there

361 were six MAPK-related genes, and these genes had higher levels of expression in YN samples

362 with high tanshinones content (Table S10), suggesting their possible role in tanshinones

363 accumulation.

364

365 Plants primary metabolism pathways may play an indirect role in secondary metabolic processes.

366 In this study, genes that involved in primary metabolic processes such as photosynthesis and

367 citrate cycle (TCA cycle) were differentially expressed only at the initial stage of tanshinones

368 accumulation (Table S7). Genes related to the regulation of circadian rhythms were specifically

369 differentially expressed at the later stage of tanshinones accumulation (Table S8). In the process

370 of plants responding to environmental changes, primary metabolism processes were affected, and

371 the fixed carbon through photosynthesis becomes allocated to secondary metabolites (Isah 2019).

372 Moreover, plants distributed energy to the secondary metabolic processes through actively

373 inhibits growth in response to environmental changes, which might sometimes involve circadian

374 rhythm response (Chaves et al. 2002; Zhang et al. 2020). These results were consistent with our

375 observations, indicating an interaction between secondary metabolism and primary metabolism. 
376 In other species, the reciprocal relationship among secondary metabolism, primary metabolism

377 and signal transduction signaling systems had also been found. For instance, DEGs in two

378 different chemotypes of Cinnamomum camphora were mainly associated with carbohydrate

379 metabolism, signal transduction, and terpenoid biosynthesis, and these biological processes-

380 related DEGs resulted in chemotype variation in C. camphora (Chen et al. 2018a). In

381 Andrographis paniculata, the biosynthesis of diterpenoid andrographolide involved the processes

382 such as citrate cycle, carbon fixation, and oxidative phosphorylation in addition to terpenoid

383 biosynthesis (Patel et al. 2020). Transcriptional studies of Daucus carota with different

384 flavonoids concentrations revealed that the accumulation of flavonoids was related to flavonoid

385 biosynthesis pathway, endogenous signaling and other secondary metabolite biosynthesis

386 pathways (Meng et al. 2020). Additionally, primary metabolism pathways such as galactose

387 metabolism (Chen et al. 2019) and starch and sucrose metabolism (Lloyd \& Zakhleniuk 2004)

388 also played an indirect role in secondary metabolism.

389

390 TFs play a predominant role in regulating the genes expression in various metabolic pathways.

391 The identification of these TFs might be critical for understanding the regulatory mechanisms of

392 tanshinones biosynthesis during tanshinones accumulation stages in different varieties of $S$.

393 miltiorrhiza. The TFs associated with tanshinones biosynthesis mainly belong to AP2/ERF

394 (Huang et al. 2019), MYB (Ding et al. 2017), bHLH (Xing et al. 2018), WRKY (Li et al. 2015)

395 and GRAS (Xing et al. 2018) family. In the present study, 24 TFs associated with tanshinones

396 accumulation were obtained, including six NAC, four AP2/ERF, three WRKY, two MYB, and

397 one MYB-related TFs. One ERF (Unigene0005368, ERF1B) and three WRKY

398 (Unigene0002677, WRKY24; Unigene0005661, WRKY22; Unigene0019306, WRKY33) TFs

399 related to the MAPK signaling pathway were identified, while one of them, WRKY33, was

400 found to possibly regulate tanshinones accumulation (Jiang et al. 2019), suggesting that the

401 possible regulatory role of the other three MAPK signaling pathway-related TFs as well as other

402 tanshinones accumulation-related (Table S10). In addition, some members of the ERP, bHLH, 
403 and MYB transcription factor families have been previously identified to play important

404 regulatory roles in tanshinone biosynthesis and accumulation. Although the NAC family has not

405 been reported in S. miltiorrhiza research, studies have identified a regulatory role for NAC

406 transcription factors in terpene biosynthetic pathways (Jeena et al. 2017; Nieuwenhuizen et al.

407 2015).

408

409 Transcriptome analysis of different varieties of $S$. miltiorrhiza under the same environment is

410 helpful to evaluate the intraspecific variation of S. miltiorrhiza. During tanshinones accumulation

411 period, 23,369 (44.75\%) of genes were differentially expressed among different varieties of $S$.

412 miltiorrhiza, indicating that there were obvious gene regulation differences among different

413 varieties of $S$. miltiorrhiza. Based on the KEGG pathway enrichment results, a total of 48 co-

414 enriched pathways were observed in each paired comparison group during the tanshinones

415 accumulation period. These common pathways of enrichment involve many physiological

416 processes. For example, "Biosynthesis of amino acids", “carbon metabolism”, "galactose

417 metabolism", and "alanine, aspartate and glutamate metabolism" are related to the basic

418 metabolic activities of plants, "plant hormone signal transduction" and "MAPK signal pathway"

419 are related to signal transduction, as well as to the biosynthesis of secondary metabolites such as

420 phenylpropanoid and flavonoid. These functional genes might contribute to intraspecific

421 variations. Among all paired comparison groups, the lowest number of DEGs genes was found

422 between $\mathrm{HN}$ and SC varieties, which might be due to a relatively small variation within these

423 two varieties, while correlation analysis between varieties based on all expressed genes also

424 showed a high correlation between $\mathrm{HN}$ and SC samples (Fig. 2, Fig. S2). Transcriptome

425 sequencing provides a wealth of biological information that has been used to differentiate

426 germplasm in crops such as maize (Frisch et al. 2010). Notably, a total of 210 (75.81\%) of the

427 content-related genes were also expressed differently in different varieties of S. miltiorrhiza,

428 suggesting that the difference of tanshinones content in different varieties of $S$. miltiorrhiza

429 might be related to the variation of tanshinones production. This result was consistent with 
430 previous studies in other species. These studies reported that different transcription regulation 431 could distinguish different varieties of the same species, such as, Peper nigrum (Khew et al. 432 2020), chamomile (Tai et al. 2020), Cinnamomum burmannii (Yang et al. 2020), C. camphora 433 (Chen et al. 2018a), Euphorbia pulcherrima (Vilperte et al. 2019) and Auricularia auricula434 judae (Zhao et al. 2019), and the relative species (Nieuwenhuizen et al. 2015; Tai et al. 2020; 435 Wang et al. 2020).

436

\section{Conclusions}

438 In this study, a comparative transcriptome analysis of four different varieties of S. miltiorrhiza 439 during tanshinones accumulation stages was performed. We found that the dynamic change of 440 tanshinones contents in this study are consistent with the phenomenon observed in related 441 studies. By comparing four varieties of $S$. miltiorrhiza, it was found that there were significant 442 transcriptome differences among varieties in the process of tanshinone accumulation, which may 443 reflect the intraspecific variation of $S$. miltiorrhiza. The functional characterization of genes 444 related to tanshinones accumulation indicated that primary metabolism and signal transduction 445 pathway might play an indirect role in tanshinones accumulation. These studies give us a better 446 understanding of the relationship between primary metabolism, signal transduction and 447 secondary metabolism, and provide a research and application basis for better regulating the 448 overall metabolism of medicinal plants to improve the biosynthesis and accumulation of 449 secondary metabolites. This has also led to a deeper understanding of intraspecific variation in 450 gene regulation in the biosynthetic pathways of plant secondary metabolites.

451

\section{Acknowledgments}

453 This study was funded by the Natural Science Foundation of China (81973416), and the 454 Chengdu University of Traditional Chinese Medicine characteristic innovative research team 455 project (CXTD2018017). 


\section{References}

458 Adachi H, Nakano T, Miyagawa N, Ishihama N, Yoshioka M, Katou Y, Yaeno T, Shirasu K, and

459

460

461

462

463

464

465

466

467

468

469

470

471

472

473

474

475

476

477

478

479

480

481

482

483

484

485

486

487

488

489

490

491

492

493

494 Yoshioka H. 2015. WRKY Transcription Factors Phosphorylated by MAPK Regulate a Plant Immune NADPH Oxidase in Nicotiana benthamiana. Plant Cell 27:2645-2663. 10.1105/tpc.15.00213

Chang Y, Wang M, Li J, and Lu S. 2019. Transcriptomic analysis reveals potential genes involved in tanshinone biosynthesis in Salvia miltiorrhiza. Scientific Reports 9:14929. 10.1038/s41598-019-51535-9

Chaves MM, Pereira JS, Maroco J, Rodrigues ML, Ricardo CP, Osório ML, Carvalho I, Faria T, and Pinheiro C. 2002. How plants cope with water stress in the field. Photosynthesis and growth. Ann Bot 89 Spec No:907-916. 10.1093/aob/mcf105

Chen C, Zheng Y, Zhong Y, Wu Y, Li Z, Xu LA, and Xu M. 2018a. Transcriptome analysis and identification of genes related to terpenoid biosynthesis in Cinnamomum camphora. BMC Genomics 19:550. 10.1186/s12864-018-4941-1

Chen S, Zhou Y, Chen Y, and Gu J. 2018b. fastp: an ultra-fast all-in-one FASTQ preprocessor. Bioinformatics 34:i884-i890. 10.1093/bioinformatics/bty560

Chen Y, Wang Y, Liu M, Qu J, Yao M, Li B, Ding M, Liu H, Xiao W, and Yuan Y. 2019. Primary and Secondary Metabolic Effects of a Key Gene Deletion ( $\triangle$ YPL062W) in Metabolically Engineered Terpenoid-Producing Saccharomyces cerevisiae. Appl Environ Microbiol 85. 10.1128/aem.01990-18

Contreras A, Leroy B, Mariage PA, and Wattiez R. 2019. Proteomic analysis reveals novel insights into tanshinones biosynthesis in Salvia miltiorrhiza hairy roots. Sci Rep 9:5768. 10.1038/s41598-019-42164-3

Dan MX, Feng CY, Yun CY, and Jing L. 2019. Danshen: a phytological overview. Chinese Journal of Natural Medicines. 10.3724/SP.J.1009.2019.00059

De Boer K, Tilleman S, Pauwels L, Vanden Bossche R, De Sutter V, Vanderhaeghen R, Hilson P, Hamill JD, and Goossens A. 2011. APETALA2/ETHYLENE RESPONSE FACTOR and basic helix-loop-helix tobacco transcription factors cooperatively mediate jasmonateelicited nicotine biosynthesis. Plant J 66:1053-1065. 10.1111/j.1365-313X.2011.04566.x

Deng KJ, Zhang Y, Xiong BQ, Peng JH, Zhang T, Zhao XN, and Ren ZL. 2009. [Identification, characterization and utilization of simple sequence repeat markers derived from Salvia miltiorrhiza expressed sequence tags]. Yao Xue Xue Bao 44:1165-1172.

Devendrakumar KT, Li X, and Zhang YL. 2018. MAP kinase signalling: interplays between plant PAMP- and effector-triggered immunity. Cellular and Molecular Life Sciences 75:29812989.

Ding K, Pei TL, Bai ZQ, Jia YY, Ma PD, and Liang ZS. 2017. SmMYB36, a Novel R2R3-MYB Transcription Factor, Enhances Tanshinone Accumulation and Decreases Phenolic Acid Content in Salvia miltiorrhiza Hairy Roots. Scientific Reports 7. 
495 Fabriki-Ourang S, and Karimi H. 2019. Assessment of genetic diversity and relationships among

496

497

498

499

500

501

502

503

504

505

506

507

508

509

510

511

512

513

514

515

516

517

518

519

520

521

522

523

524

525

526

527

528

529

530

531

532

533

534

535 Salvia species using gene targeted CAAT box-derived polymorphism markers. J Genet 98.

Fernie AR, Carrari F, and Sweetlove LJ. 2004. Respiratory metabolism: glycolysis, the TCA cycle and mitochondrial electron transport. Curr Opin Plant Biol 7:254-261. 10.1016/j.pbi.2004.03.007

Frisch M, Thiemann A, Fu J, Schrag TA, Scholten S, and Melchinger AE. 2010. Transcriptomebased distance measures for grouping of germplasm and prediction of hybrid performance in maize. Theoretical and Applied Genetics 120:441-450. 10.1007/s00122009-1204-1

Gao W, Sun HX, Xiao H, Cui G, Hillwig ML, Jackson A, Wang X, Shen Y, Zhao N, Zhang L, Wang XJ, Peters RJ, and Huang L. 2014. Combining metabolomics and transcriptomics to characterize tanshinone biosynthesis in Salvia miltiorrhiza. BMC Genomics 15:73. 10.1186/1471-2164-15-73

Ge Q, Zhang Y, Hua W-P, Wu Y-C, Jin X-X, Song S-H, and Wang Z-Z. 2015. Combination of transcriptomic and metabolomic analyses reveals a JAZ repressor in the jasmonate signaling pathway of Salvia miltiorrhiza. Scientific Reports 5:14048-14048. 10.1038/srep14048

Grabherr MG, Haas BJ, Yassour M, Levin JZ, Thompson DA, Amit I, Adiconis X, Fan L, Raychowdhury R, Zeng Q, Chen Z, Mauceli E, Hacohen N, Gnirke A, Rhind N, di Palma F, Birren BW, Nusbaum C, Lindblad-Toh K, Friedman N, and Regev A. 2011. Full-length transcriptome assembly from RNA-Seq data without a reference genome. Nat Biotechnol 29:644-652. 10.1038/nbt.1883

He Y, and Meng X. 2020. MAPK Signaling: Emerging Roles in Lateral Root Formation. Trends in Plant Science 25:126-129. 10.1016/j.tplants.2019.11.006

Huang Q, Sun MH, Yuan TP, Wang Y, Shi M, Lu SJ, Tang BP, Pan JX, Wang Y, and Kai GY. 2019. The AP2/ERF transcription factor SmERF1L1 regulates the biosynthesis of tanshinones and phenolic acids in Salvia miltiorrhiza. Food Chemistry 274:368-375.

Isah T. 2019. Stress and defense responses in plant secondary metabolites production. Biological Research 52. 10.1186/s40659-019-0246-3

Jeena GS, Fatima S, Tripathi P, Upadhyay S, and Shukla RK. 2017. Comparative transcriptome analysis of shoot and root tissue of Bacopa monnieri identifies potential genes related to triterpenoid saponin biosynthesis. BMC Genomics 18.

Jiang T, Zhang M, Wen C, Xie X, Tian W, Wen S, Lu R, and Liu L. 2020. Integrated metabolomic and transcriptomic analysis of the anthocyanin regulatory networks in Salvia miltiorrhiza Bge. flowers. BMC plant biology 20:349. 10.1186/s12870-020-025537

Jiang Y, Wang L, Lu S, Xue Y, Wei X, Lu J, and Zhang Y. 2019. Transcriptome sequencing of Salvia miltiorrhiza after infection by its endophytic fungi and identification of genes related to tanshinone biosynthesis. Pharm Biol 57:760-769. 10.1080/13880209.2019.1680706 
536 Jin JP, Zhang H, Kong L, Gao G, and Luo JC. 2014. PlantTFDB 3.0: a portal for the functional

537

538

539

540

541

542

543

544

545

546

547

548

549

550

551

552

553

554

555

556

557

558

559

560

561

562

563

564

565

566

567

568

569

570

571

572

573

574

575

576 and evolutionary study of plant transcription factors. Nucleic Acids Research 42:D1182D1187. 10.1093/nar/gkt1016

Kalapos B, Hlavova M, Nadai TV, Galiba G, Bisova K, and Doczi R. 2019. Early Evolution of the Mitogen-Activated Protein Kinase Family in the Plant Kingdom. Scientific Reports 9.

Khew CY, Harikrishna JA, Wee WY, Lau ET, and Hwang SS. 2020. Transcriptional Sequencing and Gene Expression Analysis of Various Genes in Fruit Development of Three Different Black Pepper (Piper nigrum L.) Varieties. Int J Genomics 2020:1540915. $10.1155 / 2020 / 1540915$

Li B, and Dewey CN. 2011. RSEM: accurate transcript quantification from RNA-Seq data with or without a reference genome. BMC Bioinformatics 12.

Li C, Li D, Shao F, and Lu S. 2015. Molecular cloning and expression analysis of WRKY transcription factor genes in Salvia miltiorrhiza. BMC Genomics 16:200. 10.1186/s12864-015-1411-x

Li Y, Kong D, Fu Y, Sussman MR, and Wu H. 2020. The effect of developmental and environmental factors on secondary metabolites in medicinal plants. Plant Physiol Biochem 148:80-89. 10.1016/j.plaphy.2020.01.006

Liang ZS, Liu WT, Feng XF and Cui GH, "Biological Characters of Danshen," in Dan Shen (Salvia miltiorrhiza) in Medicine, X. Yan, Ed., chapter 3, pp. 20-26, Springer, Dordrecht, The Netherlands, 2015.

Lloyd JC, and Zakhleniuk OV. 2004. Responses of primary and secondary metabolism to sugar accumulation revealed by microarray expression analysis of the Arabidopsis mutant, pho3. J Exp Bot 55:1221-1230. 10.1093/jxb/erh143

Love MI, Huber W, and Anders S. 2014. Moderated estimation of fold change and dispersion for RNA-seq data with DESeq2. Genome Biology 15.

Ma X-HM, Y.; Tang, J.-F.; . 2015. The Biosynthetic Pathways of Tanshinones and Phenolic Acids in Salvia miltiorrhiza. Molecules 20:16235-16254. 10.3390/molecules200916235

Mao G, Meng X, Liu Y, Zheng Z, Chen Z, and Zhang S. 2011. Phosphorylation of a WRKY transcription factor by two pathogen-responsive MAPKs drives phytoalexin biosynthesis in Arabidopsis. The Plant cell 23:1639-1653. 10.1105/tpc.111.084996

Meng G, Clausen SK, and Rasmussen SK. 2020. Transcriptome Analysis Reveals Candidate Genes Related to Anthocyanin Biosynthesis in Different Carrot Genotypes and Tissues. Plants (Basel) 9. 10.3390/plants9030344

Nieuwenhuizen NJ, Chen XY, Wang MY, Matich AJ, Perez RL, Allan AC, Green SA, and Atkinson RG. 2015. Natural Variation in Monoterpene Synthesis in Kiwifruit: Transcriptional Regulation of Terpene Synthases by NAC and ETHYLENEINSENSITIVE3-Like Transcription Factors. Plant Physiology 167:1243-U1198.

Patel AA, Shukla YM, Kumar S, Sakure AA, Parekh MJ, and Zala HN. 2020. Transcriptome analysis for molecular landscaping of genes controlling diterpene andrographolide biosynthesis in Andrographis paniculata (Burm . f.) Nees. 3 Biotech 10:512. 10.1007/s13205-020-02511-y 
577 Song Z, Guo L, Liu T, Lin C, Wang J, and Li X. 2017. Comparative RNA-Sequence

$578 \quad$ Transcriptome Analysis of Phenolic Acid Metabolism in Salvia miltiorrhiza, a Traditional

$579 \quad$ Chinese Medicine Model Plant. Int J Genomics 2017:9364594. 10.1155/2017/9364594

580 Song Z, Li X, Wang H, and Wang J. 2010. Genetic diversity and population structure of Salvia

581 miltiorrhiza Bge in China revealed by ISSR and SRAP. Genetica 138:241-249.

$582 \quad 10.1007 / s 10709-009-9416-5$

583 Song Z, Lin C, Xing P, Fen Y, Jin H, Zhou C, Gu YQ, Wang J, and Li X. 2020. A high-quality

584

585

586

587

588

589

590

591

592

593

594

595

596

597

598

599

600

601

602

603

604

605

606

607

608

609

610

611

612

613

614

615

616 reference genome sequence of Salvia miltiorrhiza provides insights into tanshinone synthesis in its red rhizomes. Plant Genome 13:e20041. 10.1002/tpg2.20041

Sunar S, Korkmaz M, SiĞmaz B, and AĞar G. 2020. Determination of the Genetic Relationships Among Salvia Species by RAPD and ISSR Analyses. Turk J Pharm Sci 17:480-485. 10.4274/tjps.galenos.2018.24572

Tai YL, Hou XJ, Liu C, Sun JM, Guo CX, Su L, Jiang W, Ling CC, Wang CX, Wang HH, Pan GF, Si XY, and Yuan Y. 2020. Phytochemical and comparative transcriptome analyses reveal different regulatory mechanisms in the terpenoid biosynthesis pathways between Matricaria recutita L. and Chamaemelum nobile L. BMC Genomics 21.

Thiriet-Rupert S, Carrier G, Chenais B, Trottier C, Bougaran G, Cadoret JP, Schoefs B, and Saint-Jean B. 2016. Transcription factors in microalgae: genome-wide prediction and comparative analysis. BMC Genomics 17.

Vilperte V, Lucaciu CR, Halbwirth H, Boehm R, Rattei T, and Debener T. 2019. Hybrid de novo transcriptome assembly of poinsettia (Euphorbia pulcherrima Willd. Ex Klotsch) bracts. BMC Genomics 20:900. 10.1186/s12864-019-6247-3

Wang B, Zhang Y, Chen CB, Li XL, Chen RY, and Chen L. 2007. Analysis on genetic diversity of different Salvia miltiorrhiza geographical populations in China. China journal of Chinese materia medica 32:1988-1991.

Wang C, Dong Y, Zhu L, Wang L, Yan L, Wang M, Zhu Q, Nan X, Li Y, and Li J. 2020. Comparative transcriptome analysis of two contrasting wolfberry genotypes during fruit development and ripening and characterization of the LrMYB1 transcription factor that regulates flavonoid biosynthesis. BMC Genomics 21:295. 10.1186/s12864-020-6663-4

Wang X, Zhou X, Gao W, Cui G, Huang L, and Liu C. 2011. New analysis of EST-SSR distribution and development of EST-SSR markers in Salvia miltiorrhiza. China journal of Chinese materia medica 36:289-293.

Xie Y, Ding M, Zhang B, Yang J, Pei T, Ma P, and Dong J. 2020. Genome-wide characterization and expression profiling of MAPK cascade genes in Salvia miltiorrhiza reveals the function of SmMAPK3 and SmMAPK1 in secondary metabolism. BMC Genomics 21:630. 10.1186/s12864-020-07023-w

Xing B, Liang L, Liu L, Hou Z, Yang D, Yan K, Zhang X, and Liang Z. 2018. Overexpression of SmbHLH148 induced biosynthesis of tanshinones as well as phenolic acids in Salvia miltiorrhiza hairy roots. Plant Cell Reports 37:1681-1692. 10.1007/s00299-018-2339-9

617

Xu H, Song J, Luo H, Zhang Y, Li Q, Zhu Y, Xu J, Li Y, Song C, Wang B, Sun W, Shen G, Zhang X, Qian J, Ji A, Xu Z, Luo X, He L, Li C, and Chen S. 2016. Analysis of the 
618

619

620

621

622

623

624

625

626

627

628

629

630

631

632

633

634

635

636

637

638

639

640

641

642

643

644

645

646

647

648

649

650

651

652

653

654

655

656

657

658

Genome Sequence of the Medicinal Plant Salvia miltiorrhiza. Molecular Plant 9. 10.1016/j.molp.2016.03.010

Yang L, Ding G, Lin H, Cheng H, Kong Y, Wei Y, Fang X, Liu R, Wang L, Chen X, and Yang C. 2013. Transcriptome analysis of medicinal plant Salvia miltiorrhiza and identification of genes related to tanshinone biosynthesis. PloS one 8:e80464. 10.1371/journal.pone.0080464

Yang L, Wen KS, Ruan X, Zhao YX, Wei F, and Wang Q. 2018. Response of Plant Secondary Metabolites to Environmental Factors. Molecules 23. 10.3390/molecules23040762

Yang Z, An W, Liu S, Huang Y, Xie C, Huang S, and Zheng X. 2020. Mining of candidate genes involved in the biosynthesis of dextrorotatory borneol in Cinnamomum burmannii by transcriptomic analysis on three chemotypes. PeerJ 8:e9311. 10.7717/peerj.9311

Yuan J, Zhang W, Sun K, Tang MJ, Chen PX, Li X, and Dai CC. 2019. Comparative Transcriptomics and Proteomics of Atractylodes lancea in Response to Endophytic Fungus Gilmaniella sp. AL12 Reveals Regulation in Plant Metabolism. Frontiers in Microbiology 10.

Zhan Z, Fang W, Ma X, Chen T, Cui G, Ma Y, Kang L, Nan T, Lin H, Tang J, Zhang Y, Lai C, Ren Z, Wang Y, Zhao Y, Shen Y, Wang L, Zeng W, Guo J, and Huang L. 2019. Metabolome and transcriptome analyses reveal quality change in the orange-rooted Salvia miltiorrhiza (Danshen) from cultivated field. Chin Med 14:42. 10.1186/s13020019-0265-6

Zhang G, Tian Y, Zhang J, Shu L, Yang S, Wang W, Sheng J, Dong Y, and Chen W. 2015. Hybrid de novo genome assembly of the Chinese herbal plant danshen (Salvia miltiorrhiza Bunge). Gigascience 4:62. 10.1186/s13742-015-0104-3

Zhang H, Zhao Y, and Zhu JK. 2020. Thriving under Stress: How Plants Balance Growth and the Stress Response. Developmental Cell 55:529-543.

Zhang MM, Su JB, Zhang Y, Xu J, and Zhang SQ. 2018. Conveying endogenous and exogenous signals: MAPK cascades in plant growth and defense. Current Opinion in Plant Biology 45:1-10. 10.1016/j.pbi.2018.04.012

Zhang XZ, Qian SS, Zhang YJ, and Wang RQ. 2016. Salvia miltiorrhiza: A source for antiAlzheimer's disease drugs. Pharmaceutical Biology 54:18-24.

Zhao Q, Song Z, Fang X, Pan Y, Guo L, Liu T, and Wang J. 2016. Effect of Genotype and Environment on Salvia miltiorrhiza Roots Using LC/MS-Based Metabolomics. Molecules 21:414. 10.3390/molecules21040414

Zhao Y, Wang L, Zhang D, Li R, Cheng T, Zhang Y, Liu X, Wong G, Tang Y, Wang H, and Gao S. 2019. Comparative transcriptome analysis reveals relationship of three major domesticated varieties of Auricularia auricula-judae. Sci Rep 9:78. 10.1038/s41598-01836984-y

Zheng Y, Wang P, Chen X, Sun Y, Yue C, and Ye N. 2019. Transcriptome and Metabolite Profiling Reveal Novel Insights into Volatile Heterosis in the Tea Plant (Camellia Sinensis). Molecules 24. 10.3390/molecules24183380

Peer) reviewing PDF | (2021:03:58666:4:0:NEW 10 Sep 2021) 


\section{Figure 1}

Tanshinones content in four varieties of $S$. miltiorrhiza at the different stages of tanshinone accumulation.

The $\mathrm{X}$-axis represents four different varieties in two stages, and the $\mathrm{Y}$-axis represents the content of the tanshinone $(\mathrm{mg} / \mathrm{g})$. Abbreviations for the four varieties are as follows: SC (Sichuan), SX (Shanxi), YN (Yunnan) and HN (Henan). The number 1 and 2 after the abbreviations for the varieties represent the early stage of tanshinone accumulation ( 2 days post-anthesis) and the late stage of tanshinone accumulation (60 days post-anthesis), respectively. Abbreviations for the 6 tanshinones are as follows: TNI (tanshinone I), TNIIA (tanshinone IIA), CTN (cryptotanshinone), TNIIB (tanshinone IIB), MTN (miltrinoe), DTNI (dihydrotanshinone I), and TNI (tanshinone I). 


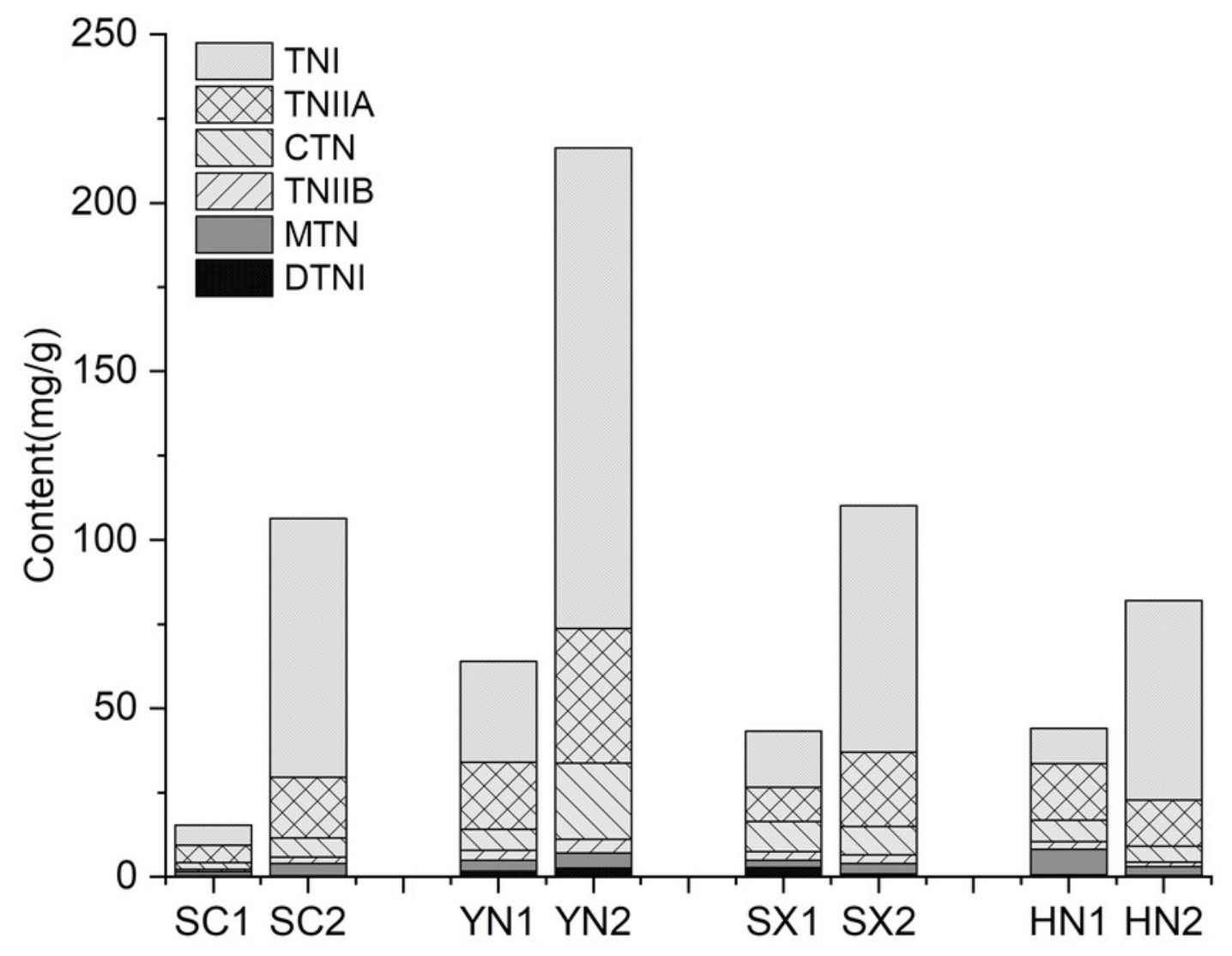


Figure 2

The summary of gene differential expression among different varieties at the early stage (S1) and late stage (S2) of tanshinones accumulation.

(A) Number of up-regulated and down-regulated DEGs in each pairwise comparison groups at stage S1. (B) Number of up-regulated and down-regulated DEGs in each pairwise comparison groups at stage S2. Abbreviations for the four varieties are as in Fig1.

A

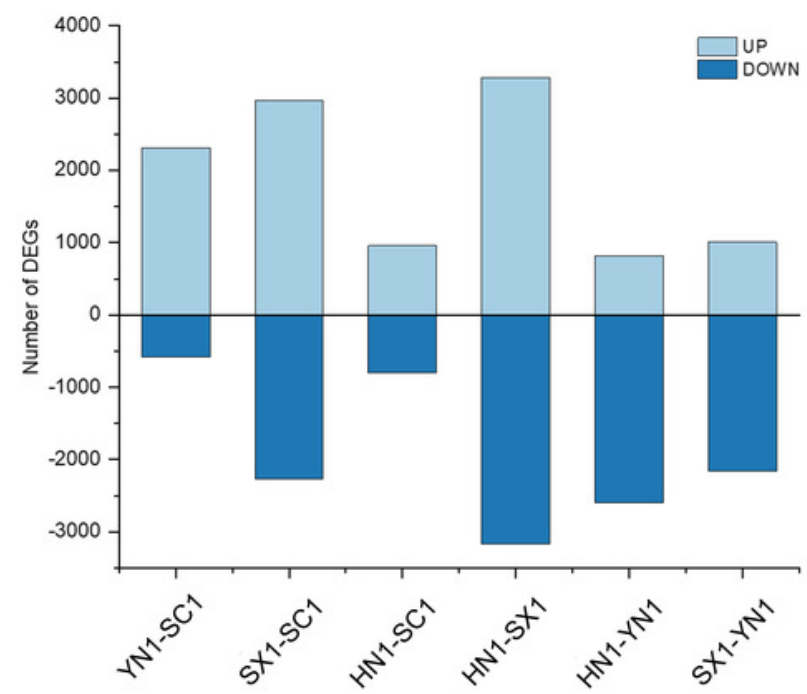

B

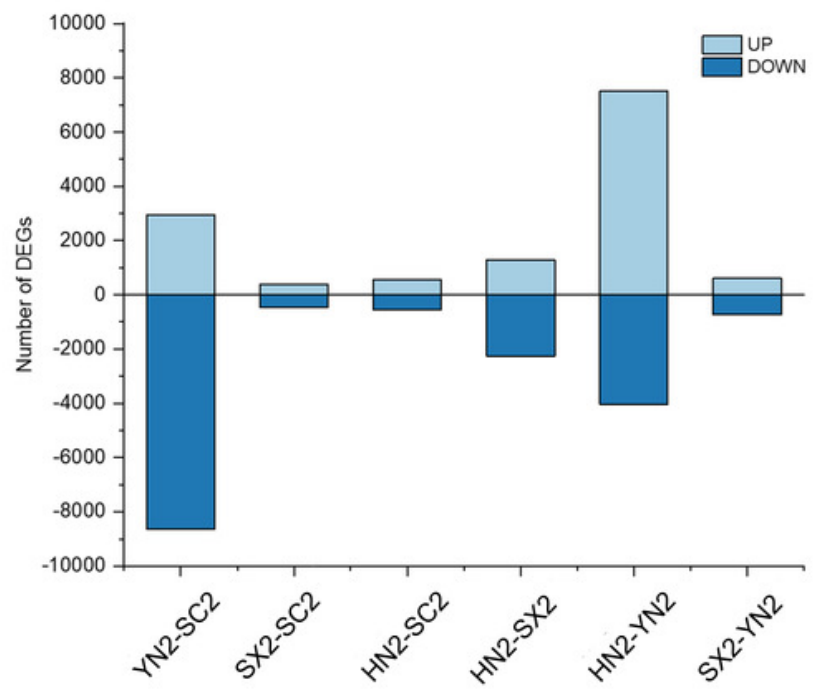




\section{Figure 3}

Differential expression analysis of four varieties of $S$. miltiorrhiza at two stages during tanshinone accumulation.

(A) Total DEGs in the four pairwise comparison group (SC1-SC2, YN1-YN2, SX1-SX2, and HN1HN2) summarized in a Venn diagram. (B) Overview of up-regulated and down-regulated DEGs of the 4 varieties of $S$. miltiorrhiza at two stages during tanshinone accumulation. (C). Heatmap of the expression levels of 2016 DEGs. (D) Pathway enrichment analysis of 2016 DEGs. (E) Pathway enrichment analysis of 277 DEGs related to tanshinone accumulation. (F) Heatmap of the expression levels of 24 differentially expressed TFs. Abbreviations for the four varieties are as in Fig1. 


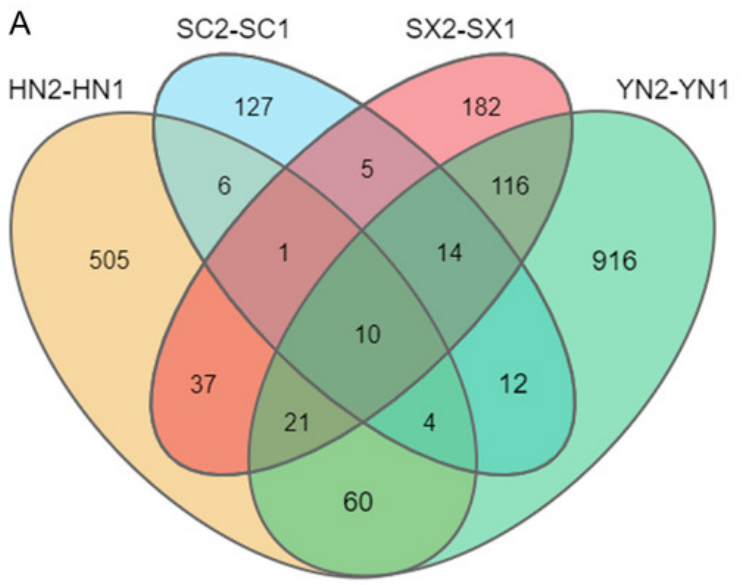

B
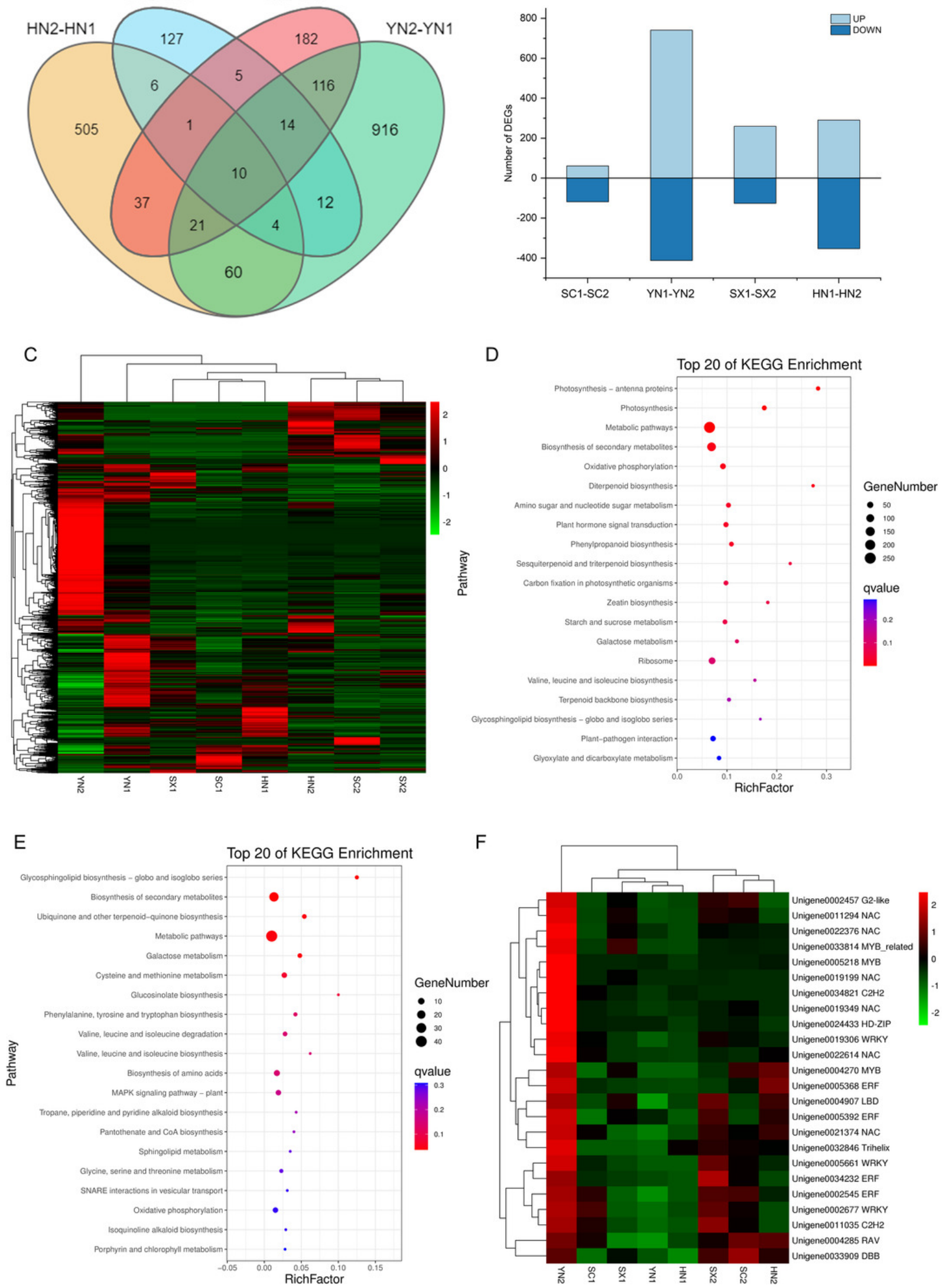
Figure 4

Correlations of transcript levels of DEGs between RNA-seq and qPCR data at stage S1 and S2.

(A) The correlation of qRT-PCR (X-axis) and RNA-seq (Y-axis) at stage S1. (B) The correlation of qRT-PCR (X-axis) and RNA-seq (Y-axis) at stage S2.
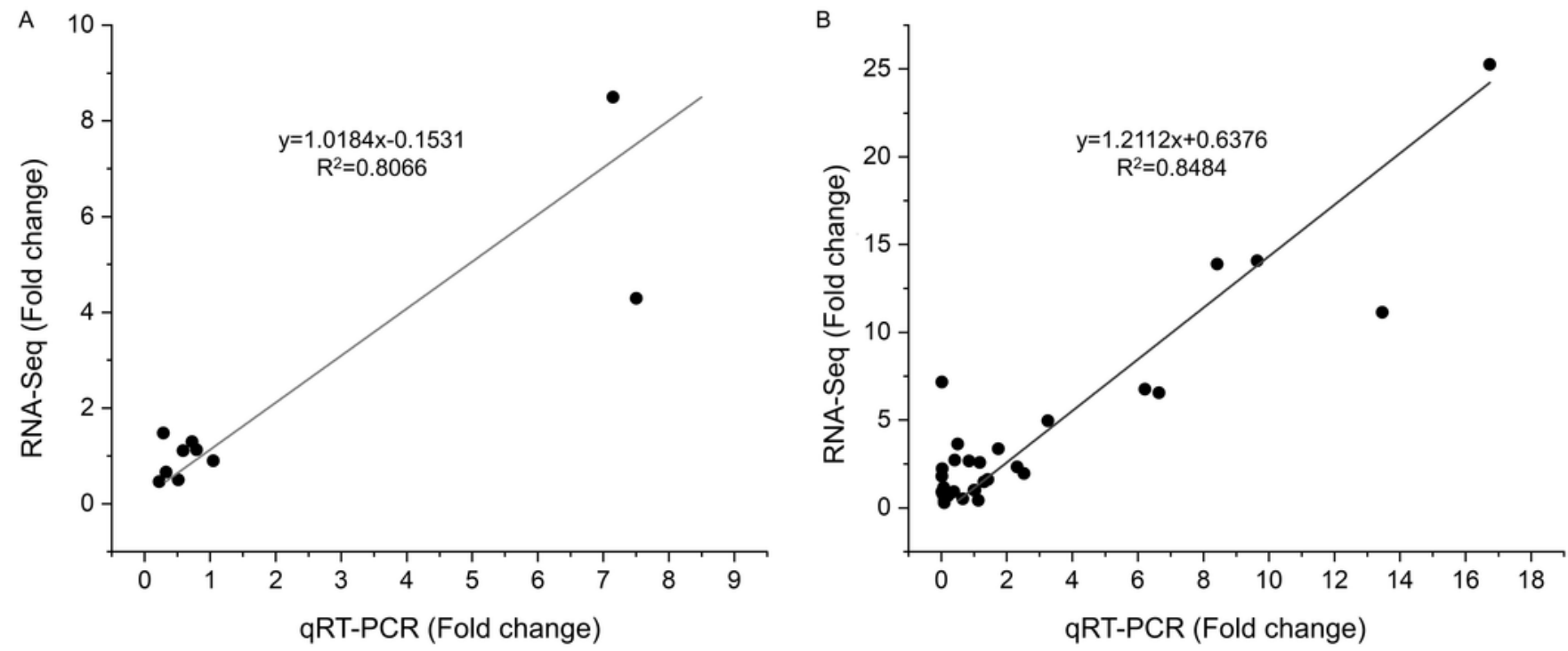


\section{Table $\mathbf{1}$ (on next page)}

Environmental information for the origin of the varieties

Notes: Abbreviations for the four varieties are as follows: SC (Sichuan), SX (Shanxi), YN (Yunnan) and HN (Henan). 
Table 1. Environmental information for the origin of the varieties.

\begin{tabular}{|c|c|c|c|c|c|c|}
\hline Group & Declaration of Origin & $\begin{array}{l}\text { Altitude } \\
\text { (m) }\end{array}$ & $\begin{array}{l}\text { Latitude } \\
\left(^{\circ}\right)\end{array}$ & Longitude $\left(^{\circ}\right)$ & $\begin{array}{c}\text { Mean annual temperatures } \\
\left({ }^{\circ} \mathrm{C}\right)\end{array}$ & Mean Annual Precipitation (mm) \\
\hline $\mathrm{SC}$ & Deyang city, Sichuan Province & 784 & 31.01 & 105 & 16.7 & 883 \\
\hline $\mathrm{YN}$ & Mile city, Yunnan Province & 1792 & 24.59 & 103.62 & 17.3 & 987.5 \\
\hline SX & Tongguan city, Shanxi Province & 330 & 34.62 & 110.18 & 12.8 & 625.5 \\
\hline $\mathrm{HN}$ & Sanmenxia city, Henan Province & 673 & 32.61 & 111.96 & 12.5 & 848 \\
\hline
\end{tabular}

Notes: Abbreviations for the 4 varieties are as follows: SC (Sichuan), SX (Shanxi), YN (Yunnan) and HN (Henan). 


\section{Table 2 (on next page)}

Number of DEGs in each comparison group at two tanshinones accumulication stages

Notes: The number of differentially expressed genes among varieties at S1 stage was shown at the bottom left of the table. The number of differentially expressed genes among the varieties at S2 stage was shown in the upper right. Abbreviations for the four varieties are as follows: SC (Sichuan), SX (Shanxi), YN (Yunnan) and HN (Henan). 
Table 2. Number of DEGs in each comparison group at two tanshinones accumulication stages

\begin{tabular}{ccccc}
\hline & SC & YN & HN & SX \\
\hline SC & - & 11603 & 1763 & 853 \\
YN & 2888 & - & 11563 & 1330 \\
HN & 1119 & 3417 & - & 3542 \\
SX & 5236 & 3167 & 6448 & - \\
\hline
\end{tabular}

Notes: The number of differentially expressed genes among varieties at S1 stage was shown at the bottom left of the table. The number of differentially expressed genes among the varieties at S2 stage was shown in the upper right. Abbreviations for the 4 varieties are as follows: SC (Sichuan), SX (Shanxi), YN (Yunnan) and HN (Henan). 